Sacristán San Cristóbal, M., Martín R., D., Navarro Asensio, E. \& Tourón Figueroa, J. (2017). Flipped Classroom y Didáctica de las Matemáticas en la Formación online de Maestros de Educación Infantil. Revista Electrónica Interuniversitaria de Formación del Profesorado, 20 (3), 1-14. DOI: http://dx.doi.org/10.6018/reifop.20.1.292551

\title{
Flipped classroom y didáctica de las matemáticas en la formación online de maestros de Educación Infantil
}

\author{
Mara Sacristán San Cristóbal ${ }^{(1)}$, Déborah Martín R. ${ }^{(1)}$, Enrique Navarro Asencio ${ }^{(2)}$, Javier \\ Tourón Figueroa $^{(1)}$ \\ (1) Universidad Internacional de La Rioja, ${ }^{(2)}$ Universidad Complutense de Madrid
}

\section{Resumen}

El presente trabajo de investigación se ha llevado a cabo en la asignatura Didáctica de las Matemáticas en la formación de maestros de Educación Infantil, siguiendo el modelo pedagógico Flipped Learning, en un entorno completamente online. Este modelo compromete a invertir los roles del docente y del alumno, haciendo a este último protagonista de su propio proceso de aprendizaje.

El objetivo ha sido analizar los resultados de aprendizaje y la satisfacción del alumnado tras experimentar el modelo flipped classroom en uno de los temas de la asignatura. Para ello, se utilizó un diseño cuasiexperimental con un grupo de control no equivalente y una medida pretest de los resultados de aprendizaje para controlar las posibles diferencias iniciales entre los grupos.

Se explicitan las distintas fases en la aplicación del modelo flipped, tanto la preparación previa a la sesión presencial virtual del profesor y los alumnos, como el desarrollo de la misma y la evaluación final.

Los resultados de este estudio muestran que este enfoque pedagógico aumenta el rendimiento de los alumnos y más aún cuando se asiste en directo a las sesiones. Igualmente, las valoraciones subjetivas obtenidas tienen como eje común la motivación y satisfacción hacia la metodología experimentada.

\section{Palabras clave}

Clase inversa; Aprendizaje Inverso; Formación de maestros; Educación Online.

\section{Contacto:}

Javier Tourón. Vicerrector de Innovación y Desarrollo Educativo. Universidad Internacional de La Rioja. c/ Avenida de la Paz, 137, 26006 Logroño (La Rioja). Telf. 941210211 ext. 1388. javier.touron@unir.net 


\title{
Flipped classroom and mathematics didactics in online education of Early Childhood Teachers
}

\begin{abstract}
This research work has been carried out in the subject Didáctica de las Matemáticas in the training of teachers of Early Childhood Education following the pedagogical model Flipped Learning in a completely online environment. This model commits to reverse the roles of the teacher and students making the latter the protagonists of their own learning process.

The objective was to analyse the learning outcomes and student satisfaction after experiencing the flipped classroom model in one of the units of the subject. For this, a quasiexperimental design was used with a non-equivalent control group and a pretest measure of learning outcomes to control possible initial differences between groups.

The different phases in the application of the flipped model are explained, both the preparation before the virtual face-to-face session of the teacher and the students, and its development and the final evaluation.

The results of this study show that this pedagogical approach increases student performance and even more when they attend live sessions. Likewise, the subjective assessments obtained have as a common axis: the motivation and satisfaction towards the methodology experienced.
\end{abstract}

\section{Key words}

Flipped Classroom; Flipped Learning; Teacher Training; Online Education

\section{Introducción}

El despliegue del Espacio Europeo de Educación Superior (EEES) ha supuesto un modelo educativo enfocado al desarrollo de competencias. Estas son entendidas como el aprendizaje de un conjunto de conocimientos, destrezas, habilidades y actitudes que deben aplicarse y transferirse a un contexto determinado.

Esta orientación metodológica, dirigida al desarrollo competencial, traslada un modelo universitario centrado en la enseñanza, a un modelo centrado en el aprendizaje (Rué, 2007). Por otra parte, el Sistema de Transferencia y Acumulación de Créditos (ECTS) implica el cómputo de horas de clases lectivas, horas de estudio, horas dedicadas a la realización de seminarios, trabajos, prácticas o proyectos, y las exigidas para la preparación, y realización de las pruebas de evaluación, para la obtención de créditos, lo que sitúa el aprendizaje en su condición global (Valiente, Kindelan, Ayuga y Martín, 2016)

Este enfoque requiere un modelo pedagógico centrado en el aprendizaje (Tourón y Santiago, 2015), y más concretamente en la formación de los futuros profesores (Martín y Santiago, 2016).

Ante estas nuevas propuestas europeas, el modelo educativo Flipped Classroom, puede ser de los más congruentes con la filosofía del EEES (Espacio Europeo de Educación Superior). Este modelo Tourón (2016) lo define como: 
Un enfoque pedagógico y metodológico paidocéntrico que lleva a personalizar el aprendizaje de cada estudiante, ayudándole a asumir el peso y la responsabilidad de su propio progreso y desarrollo personal, haciendo para ello uso de la tecnología digital como herramienta necesaria para llevar a cabo dicha personalización, al tiempo que se fomenta un aprendizaje más profundo, flexible y creativo, de modo que el profesor se convierte en guía, mentor y consejero en el itinerario de cada alumno hacia el logro de sus metas.

Este enfoque, de acuerdo con la definición anterior, compromete a invertir los roles del docente y del alumno, haciendo a este último protagonista de su propio proceso de aprendizaje. El profesor, ahora, debe definir objetivos específicos para la instrucción, que se realiza fuera del aula, así como para las sesiones presenciales; reorganizar los contenidos y los espacios, orientándose a los modelos Blended Learning; mantener un espacio de aula versátil, donde incorporar metodologías activas y tecnología digital que facilite el proceso de enseñanza-aprendizaje. Entonces ¿qué sucede en el entorno de la formación universitaria online? Podríamos suponer que al utilizar tecnología y herramientas digitales, como medios ordinarios para la formación, se facilitaría el desarrollo competencial de los estudiantes. Sin embargo, "el hecho de realizar los estudios de forma online no garantiza que los alumnos adquieran las competencias necesarias, ni siquiera la competencia tecnológica, sino que es la labor docente de los profesores y su metodología de enseñanza la que repercutirá en dicha adquisición" (Íñigo, 2016, p.475).

Por supuesto, la tecnología y las actividades que se realicen durante las clases (sea en el entorno online o presencial) son elementos esenciales en este modelo, pero lo primordial es conocer la filosofía pedagógica que lo sustenta que es anterior al manejo de herramientas tecnológicas. Esta unión será crucial para involucrar al alumno y conseguir el éxito de su aprendizaje (Martín y Tourón, en prensa). Las herramientas no se utilizan exclusivamente para la entrega de tareas en formato digital (Rivera, Sánchez, Romo, Jaramillo y Valencia, 2013), o para acceder al aula virtual, sino para la adquisición de conocimientos, el desarrollo de destrezas y habilidades, es decir, competencias (Valero, Redondo y Palacín, 2012).

Algunos autores como (Quirk-Dorr, Anderson y Hoover, 2013) se pronuncian sobre sus ventajas (Walsh, 2013a) en un panel de expertos; además, existen investigaciones empíricas sobre su impacto (Bergmann y Sams, 2012; Calvillo, 2014; Martín y Santiago, 2015; Szoka, 2013; Walsh, 2013b), entre las que se pueden destacar:

a) Adaptación al ritmo de cada estudiante. Potencialmente, el modelo Flipped Classroom permite al profesor fijar un el contenido por semanas, meses o el curso entero, permitiendo a los estudiantes que aceleren su aprendizaje a través del plan de estudios si están listos (Tourón, Santiago y Díez, 2014).

b) Implicación del estudiante; menor estrés (Marlowe, 2012); mayor participación y desarrollo del pensamiento crítico (Martín y Núñez, 2015); mayor colaboración, formulan más preguntas (Tourón, 2015).

c) Aprendizaje más profundo: permite realizar actividades que desarrollen las categorías cognitivas de orden superior de la taxonomía de Bloom (Anderson y Krathwohl, 2001).

d) Proceso y resultados de aprendizaje (Martín y Núñez, 2015; Calvillo, 2014). Similares resultados obtienen Marcey and Brint (2011) quienes señalan que el grupo experimental (flipped classroom), obtuvo unos resultados considerablemente mejores, tanto en los cuestionarios como en el examen final. 
Ahora bien, ¿cuáles son los resultados en estudios de formación universitaria online desarrollados bajo el modelo flipped? Existen estudios en la formación presencial (Angelini y García-Carbonell, 2015; Lin, Zhu, Chen, Wang, Chen, Li y Zou, 2017; Martín R. y Núnez, 2015; Martín R. y Tourón, (en prensa); Pierce y Fox, 2012; Prieto, Díaz, Monserrat y Reyes, 2014; O'Flaherty y Phillips, 2015; Thai, De Wever y Valcke, 2017), pero todavía muy pocos estudios sobre Flipped Learning en entornos online (Galway, Corbett, Takaro, Tairyan y Frank, 2014; Marshall, 2016; Marshall y Buitrago, 2017; Sun, Wu y Lee, 2016). A priori, podemos pensar que se produce de la misma manera, el alumno tiene acceso al contenido de forma permanente en su aula virtual o Learning Management System (LMS), puede acceder a los vídeos, textos o audios, previamente a la sesión presencial virtual y utilizar éstas para aclaración de dudas, realizar actividades prácticas o reflexivas utilizando dinámicas, técnicas de aprendizaje cooperativo o colaborativo o estudios de casos, entre otros (Íñigo, 2015).

\section{Metodología}

El objetivo de este estudio es analizar los resultados de aprendizaje y la satisfacción del alumnado tras experimentar el modelo flipped classroom en el tema: "Las magnitudes en Educación Infantil", de la asignatura de Didáctica de las Matemáticas del segundo curso en el Grado de Maestro en Educación Infantil en el año académico 2016-2017. El Grado, al completo, se desarrolla en un entorno online y, por tanto, la experiencia flipped también.

La hipótesis plantea que los alumnos que reciban la instrucción con un modelo flipped obtendrán mejores resultados que los que lo hagan con el modelo convencional.

Por otra parte, se espera que los alumnos del grupo experimental muestren un grado elevado de satisfacción con la experiencia.

\section{Participantes}

Se utilizó un diseño cuasiexperimental con grupo de control no equivalente (Campbell y Stanley, 1963) y se utilizó una medida pretest de los resultados de aprendizaje para controlar las posibles diferencias iniciales. La muestra está compuesta por 64 alumnos, de los cuales 19 pertenecen al grupo experimental y 43 al grupo de control.

\section{Variables}

La variable independiente es la modalidad de intervención. Mientras que el grupo experimental utiliza el modelo flipped classroom, el grupo de control sigue el modelo expositivo tradicional (en ambos casos online).

La variable dependiente son los resultados de aprendizaje obtenidos por los alumnos en la unidad seleccionada, a partir de un trabajo práctico evaluado mediante una rúbrica, idéntica en ambos grupos.

Se trató de aumentar la validez interna de la investigación incluyendo como covariable los resultados previos de aprendizaje, calculado a partir de las calificaciones de todas las tareas anteriores de la misma materia hasta el comienzo de la situación experimental.

Tanto la variable dependiente como la covariable varían entre o y 10 puntos, aunque no se pueden comparar directamente porque son producto de la evaluación de contenidos distintos. Por ese motivo, no se analizan diferencias pretest-postest.

Otro factor que se analizó, en este caso en el grupo experimental, es posible efecto de la asistencia o no a las sesiones presenciales virtuales bajo la metodología flipped. 
Además, se ha analizado la percepción de los alumnos respecto a la mejora de su experiencia de aprendizaje a través del cuestionario de Driscoll (2012) que se incluye en el anexo y cuyos ítems se valoran con una escala tipo Likert de 5 puntos.

\section{Procedimiento: desarrollo de la experiencia}

La construcción de las nociones de magnitud y medida deben arrancar en esta etapa para que los niños entren en contacto con situaciones que les provoquen el descubrimiento de las magnitudes, a partir de sus percepciones de determinadas propiedades en los objetos (Chamorro, 2005). De ahí que, en cumplimiento con la competencia específica CE33 de esta asignatura: "Conocer los fundamentos matemáticos del currículo de esta etapa así como las teorías sobre la adquisición y desarrollo de los aprendizajes correspondientes", los objetivos definidos en este tema fueron:

- Saber qué es una magnitud.

- Saber cómo se produce su conocimiento en el niño.

- Conocer cada una de las etapas en la progresión de la enseñanza de las magnitudes.

- Analizar cómo trabajar cada una de las magnitudes en Educación Infantil.

- Diseñar actividades para el aprendizaje de las magnitudes en Educación Infantil.

Fases en la aplicación del modelo flipped a la unidad didáctica

1. Preparación previa del profesor

En primer lugar se grabaron dos vídeos de unos 8 minutos cada uno en el estudio de grabación de la Universidad. En ellos aparece la profesora explicando los contenidos sobre una presentación en formato ppt. Posteriormente, estos vídeos se enriquecieron con preguntas emergentes a través de EDPuzzle, de tal manera que el alumno podrá ir contestando a las mismas y obtener un feedback inmediato en función de sus respuestas.

\section{Antes de la sesión presencial virtual}

Tras la edición de los vídeos, el departamento de contenidos de la Universidad los insertó en el aula virtual como una parte más de este tema para que estuvieran a disposición de los alumnos una semana antes de la clase presencial virtual. Los alumnos necesitan iniciar sesión en EDPuzzle para el visionado mediante un nombre de usuario y contraseña, de tal manera que sus respuestas quedan registradas para que puedan ser revisadas por el profesor antes de la sesión presencial y así trabajar sobre aquellos contenidos que hayan entrañado mayor dificultad entre los alumnos. Los vídeos fueron visionados por 26 alumnos de 42 matriculados en la asignatura (62\%), aunque solo 10 estudiantes (24\%) lograron superar con éxito el $50 \%$ de las preguntas planteadas.

\section{Durante la sesión presencial virtual}

Los alumnos acuden a la sesión presencial virtual habiendo trabajado previamente el contenido del tema, puesto que el vídeo está preparado para que no se puedan omitir las preguntas, sino que hay que visionarlo y contestar activamente. Por tanto, en esta sesión se profundizó en aquellas cuestiones que obtuvieron peor puntuación y en otras que surgieron posteriormente como consecuencia de una toma de contacto previa del alumno con el contenido del tema antes de la clase.

Seguidamente se planteó una actividad grupal, posible de manera online a través de Adobe Connect en su función "Crear grupos de trabajo", de tal manera que la clase se divide en 
salas virtuales independientes en las que cada grupo trabaja de manera autónoma, como si estuvieran compartiendo una misma mesa colocados en otro rincón de la clase. Los grupos constaban de tres personas que la herramienta elige al azar, aunque también es posible que el profesor pueda elegir a los integrantes de los equipos según su criterio.

Cada grupo tiene comunicación directa con el profesor, el cual puede ir entrando a cada grupo para revisar el trabajo, solventar dudas o hacer cualquier comentario, igual que si nos paseáramos por las mesas de los grupos en un aula física. En cada sala, los integrantes de cada equipo se pueden comunicar entre sí por el chat, o darles acceso al micrófono para que puedan hablar. Asimismo se pueden abrir pizarras, notas o compartir documentos en los que todos colaboren con el fin de tener recopilado el trabajo que se va realizando en cada grupo.

La actividad planteada fue la elección de una determinada magnitud (longitud, capacidad, masa o tiempo) para ser trabajada en Educación Infantil, describiendo cada una de las fases descritas para su aprendizaje por parte de los niños, es decir, las cuatro primeras de las siete que se describen en la progresión de la enseñanza de la magnitudes (Chamorro, 2015, p. 324):

- Estimación sensorial. Apreciación de la magnitud

- Comparación directa (sin intermediarios)

- Comparación indirecta (uso de un intermediario)

- Elección de una unidad

Una vez finalizada esta parte de la actividad, todos los alumnos volvieron a la sala general mediante la opción "Finalizar grupos de trabajo" y se importaron a esta los documentos colaborativos para ser compartidos con toda la clase. A continuación, un miembro de cada grupo se prestó voluntario para exponer la actividad en cuestión que, a su vez, implicaba diseñar actividades apropiadas para poner en práctica en el aula de Educación Infantil. La co-evaluación de dicha actividad se realizó a través de una rúbrica con indicadores y niveles de dominio diferenciados para facilitar al alumno la información sobre su realización y evaluación, además de asumir la responsabilidad en su propio aprendizaje. Este documento se subió previamente al apartado de Documentación de la herramienta Adobe Connect, junto con los archivos PDF correspondientes a las presentaciones de los dos vídeos, e igualmente se compartió en directo en la sesión presencial virtual. De esta manera, surgió un debate junto con la profesora acerca de las actividades más fructíferas y que podrían adecuarse más a un aula de Educación Infantil, desechando otras que, si bien en un inicio podrían parecer atractivas, tras el análisis en gran grupo resultaron ser más complicadas en la práctica, algo muy valioso para la futura acción docente del alumno del Grado de Maestro. Esta actividad en sí misma constituye una de las competencias básicas descritas en la asignatura: "Diseñar y planificar propuestas de enseñanza/aprendizaje en las que se apliquen estrategias y métodos propios de la didáctica de las matemáticas".

\section{Después de la clase presencial virtual}

Tras esta sesión presencial virtual, en la asignatura se propone una actividad individual puntuable, para diseñar actividades de las etapas de la progresión didáctica de las magnitudes, que es algo que, como acabamos de describir, el alumno ya ha tenido oportunidad de trabajar con el profesor y en equipo con sus compañeros de clase. La grabación de esta sesión se puso a disposición de todo el alumnado, en el aula virtual, para su visionado en diferido para aquellas personas que no pudieron asistir al directo. 
Igualmente, para esta actividad individual, los alumnos tenían disponible la rúbrica de su evaluación en el apartado de Documentación. Los vídeos permanecieron a disposición del alumno incluso después de la experiencia Flipped para que pudieran servir como material de estudio de la asignatura de cara al examen final.

\section{Plan de análisis de datos}

Los datos de las calificaciones de las actividades individuales fueron sometidos a un análisis comparativo frente a los datos de las calificaciones de rendimiento previo de los alumnos durante el periodo lectivo anterior en la misma asignatura, desde el inicio hasta la situación experimental. El objetivo es comprobar si los grupos difieren significativamente en la variable de rendimiento previo, que se ha seleccionado por su valor predictivo alto sobre el rendimiento posterior; además nos interesa utilizarla como covariable en el análisis de las diferencias entre los grupos, para ajustar cualquier posible diferencia inicial (aunque no fuese significativa), a los resultados en la variable dependiente.

Como eje central de la investigación, se lleva a cabo una comparación de los resultados de aprendizaje en la actividad "diseño de las actividades de las etapas de la progresión didáctica de las magnitudes para el aula de educación infantil”, del grupo que trabajó bajo la metodología flipped y el grupo de control. Se aplicó un análisis de covarianza empleando como variable independiente el grupo y como covariable los resultados iniciales de aprendizaje. También se ha estimado el tamaño del efecto empleando el estadístico eta al cuadrado parcial y las diferencias en los resultados finales de los grupos sin el efecto del rendimiento previo mediante el test de Bonferroni.

La normalidad para la variable dependiente se analiza con la prueba de Kolmogorov. El grupo control obtiene una $z=.854(p=.460)$; mientras que el grupo experimental tiene una $\mathrm{z}=.657$ y $(\mathrm{p}=.781)$, ambos casos permiten aceptar la hipótesis nula.

La misma prueba de normalidad se realiza en el grupo experimental, teniendo en cuenta la asistencia a la sesión presencial o no. Los resultados en el grupo que no asiste son: $z=.49$ $(p=.969)$, y en el alumnado que asiste a las sesiones de trabajo $z=.60(p=.867)$. También se acepta la hipótesis nula.

A continuación, los resultados de aprendizaje de las actividades individuales previas a la situación experimental se compararon entre los grupos mediante una prueba t de Student para muestras independientes. El objetivo era comprobar si la muestra era homogénea respecto a los niveles de rendimiento antes del comienzo.

Al finalizar la experiencia en el grupo experimental se aplica el cuestionario de satisfacción, voluntario y anónimo (con un 33\% de participación), del que se calculan las medias obtenidas para cada ítem. El cuestionario lo cumplimentan los alumnos comparando su experiencia de aprendizaje previa a la situación experimental en esta misma asignatura.

\section{Resultados}

Existe una diferencia en el promedio de las notas a favor del grupo control, un total de 0,983 puntos (Tabla 1). Por tanto, los grupos no son equivalentes respecto a los resultados de aprendizaje previo y es necesario introducir esta variable como elemento de control, tal como se había previsto. Previo a este análisis se comprobó la homocedasticidad de los grupos mediante la prueba $F$ de Levene $(F=0,228 ; p=0,635)$, lo que permite aceptar la hipótesis nula. 
Tabla 1.

Prueba T de Student en los resultados previos grupo control y experimental.

\begin{tabular}{lccccc}
\hline GRUPO & $\mathrm{N}$ & Media & D.T. & t de Student & $P$ \\
\hline Control & 43 & 7,779 & 1,443 & 2,685 & 0,009 \\
\hline Experimental & 19 & 6,795 & 1,016 & & \\
\hline
\end{tabular}

Los resultados del análisis de covarianza para las diferencias entre los grupos, tomando como covariable el rendimiento previo $(F=12,451 ; p=0,001)$, que presenta diferencias entre los grupos experimental y control, arrojan diferencias significativas ( $F=11,029 ; p=0,002)$, con un tamaño del efecto de 0,157 como señala el estadístico eta cuadrado parcial. La diferencia de las medias ajustadas (eliminando el efecto del rendimiento previo) es 1,209 ( $p=0,002)$ a favor del grupo experimental (ver figura 1). Esta diferencia es significativa como muestra el test de Bonferroni.

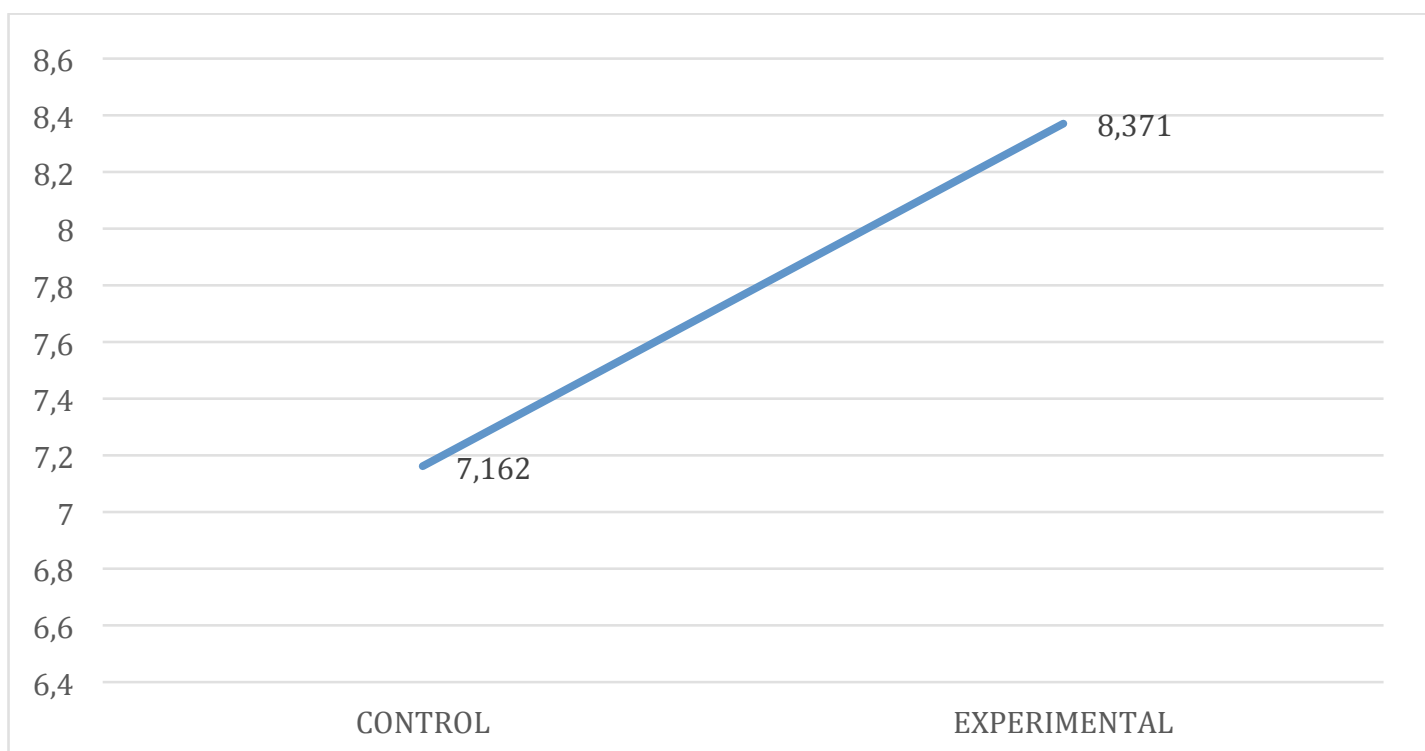

Figura 1. Valores medios de los resultados posttest del grupo control y experimental ajustando el efecto del rendimiento previo

Adicionalmente, se han analizado las diferencias de rendimiento en el grupo experimental en función de que los alumnos asistieran a las sesiones presenciales virtuales o las viesen en diferido.

Los resultados de la diferencia de medias para estos dos subgrupos (dentro del grupo experimental) reflejan un valor de 7,611 puntos para los alumnos que no asisten a las sesiones $(n=9)$ y de 8,50 para los que sí asisten $(n=10)$. Las diferencias entre ambos grupos ( 0,89 puntos), aunque aparentes desde el punto de vista práctico, no son estadísticamente significativas $(t=1,507 ; p=.158)$, dado el tamaño de los grupos.

El cuestionario de satisfacción de los alumnos con la metodología flipped ofrece los resultados que refleja la figura 2 . De la percepción de los estudiantes con la metodología experimental destacan, principalmente los siguientes aspectos: "el aprendizaje es más activo y experiencial"; "la inversión de tiempo es mayor"; "la metodología flipped les ha gustado más"; "el profesor dispone un clima facilitador"; "han podido autoevaluar su aprendizaje"; "las interacciones con el profesor son más positivas"; "más facilidad para 
acceder al material" y "ha mejorado su proceso de aprendizaje". Por el contrario, los aspectos menos valorados han sido "la expresión oral y escrita", "trabajar a mi propio ritmo" e "interacciones más frecuentes con el profesor". Esto último puede deberse a que ya de por sí, la asignatura se desarrolla con bastante participación.

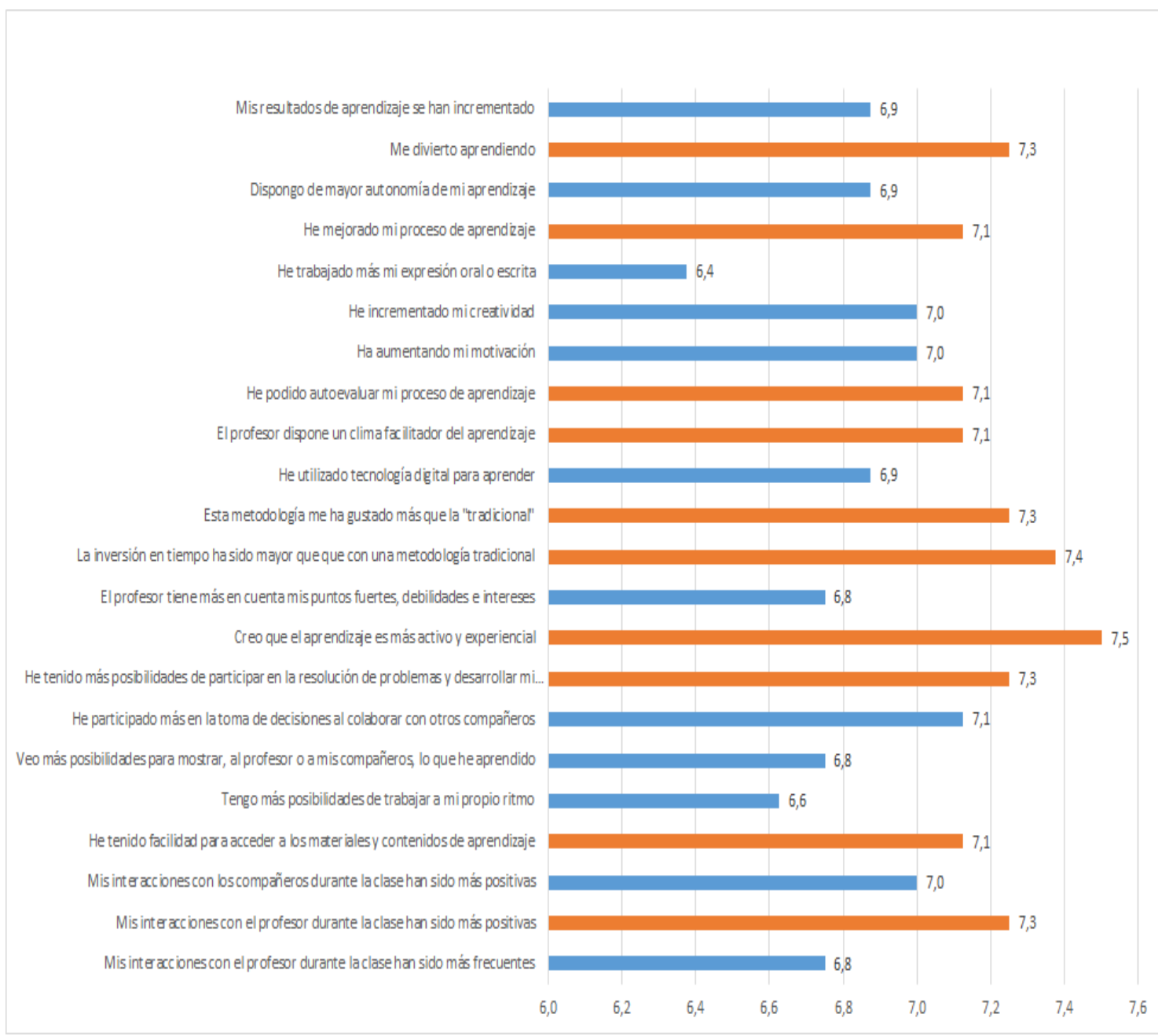

Figura 2. Resultados medios de respuesta obtenidos en el cuestionario de satisfacción de escala Likert

Por último destacamos algunas de las respuestas de los estudiantes a las preguntas abiertas del cuestionario.

- Menciona aquellos aspectos que no te han ayudado en el aprendizaje.

Al inicio la herramienta virtual no dejaba ver la clase principal cuando ya estábamos en grupos.

Con la mejora del uso de la herramienta y la habituación a usarla por parte de todos, estos momentos de incertidumbre no se darían.

- ¿Qué propuestas de mejora añadirías?

Utilizar está metodología más a menudo y en otros temas de la asignatura.

Me ha parecido fantástica la metodología, pero compartiendo los vídeos una semana y la siguiente sesión en directo para dudas y trabajo. 
Creo que se debería implementar esta metodología aunque llevará más tiempo por temas, si es viable 1 semana vídeos y la siguiente una directa porque si no, nos llevará mucho más trabajo compaginar los estudios con lo laboral y familiar.

- Escribe cualquier observación que te parezca útil para describir tu experiencia de aprendizaje, sobre la metodología o el docente y que no haya sido reflejada en las preguntas anteriores

Imagino que para quien no pudo estar en la presencial la clase al verla grabada no se aprovecha de la misma manera.

La mayoría de las clases no las pude ver en directo. Así que de alguna manera enfocaría sólo algunas clases en las que la práctica sea una muy buena tarea como la actividad de la magnitud.

Pienso que es una manera muy útil de aprender y sobre todo una manera más adecuada y por supuesto más satisfactoria de aprender y de entender mejor los contenidos.

Me habría gustado que se hubiera utilizado esta metodología en otros temas de la asignatura.

Creo que el aprendizaje es más positivo y más motivador puesto que pones en práctica los aprendizajes y te das cuenta en lo que fallas y necesitas mejorar. Muchas gracias por esa metodología, yo estoy muy agradecida porque me ayudó mucho.

Me faltaban ganas y motivación en esta asignatura y gracias a esa clase las recuperé.

\section{Discusión y conclusiones}

Los resultados de este estudio tienen una significación estadística a favor del grupo experimental, del mismo modo que la tienen práctica. En efecto, el grupo que experimenta la metodología flipped tiene mejor rendimiento, del mismo modo que los alumnos que siguen todas las sesiones en directo también mejoran su rendimiento sobre los que no lo hacen (aunque no se alcanza la significación estadística en este caso). Esto puede estar poniendo de manifiesto la importancia de seguir presencialmente las sesiones virtuales, dado que de este modo se hace más patente la bondad de la metodología. A pesar de ello, también es posible que las sesiones se aborden en diferido con cierto aprovechamiento, si bien menor que en el caso contrario.

Además de los resultados más objetivos referidos al rendimiento, no es menos importante en un estudio de caso, como el presente, la valoración de las dimensiones subjetivas llevada a cabo por los alumnos en cuanto a su motivación y satisfacción con la metodología experimentada.

Las valoraciones de los alumnos ponen de manifiesto que el aprendizaje es más activo y experiencial, el tiempo de inversión es mayor; el clima que promueve el profesor suele facilitar el aprendizaje y las interacciones con el profesor son mejores. También encuentran mayor facilidad al acceder al material, por último, valoran que su proceso de aprendizaje ha mejorado y, en general, les gusta más esta metodología. En esto los resultados presentados coinciden con las valoraciones realizadas en otros estudios (Bergmann y Sams, 2012; Calvillo, 2014; Marlowe, 2012; Martín y Núñez, 2015; Martín y Santiago, 2015; Martín y Tourón, (en prensa); Szoka, 2013; Walsh, 2013b), si bien en entornos online todavía son menos frecuentes.

Este estudio presenta las limitaciones obvias de ser un estudio de campo con grupos naturales, y llevado a cabo con una muestra limitada de alumnos, pero los resultados son 
plenamente coincidentes con los de otros autores y abundan en la superioridad de una metodología en la que el alumno ocupa un lugar mucho más activo y central. Nuevos estudios con muestras más amplias nos permitirán confirmar estos extremos.

\section{Referencias}

Anderson, L. W. y Krathwohl, D. R. (Eds.). (2001). A Taxonomy for Learning, Teaching, and Assessing: A Revision of Bloom's Taxonomy of Educational Objectives. New York: Longman.

Angelini, M. L. y García-Carbonell, A. (2015). Percepciones sobre la Integración de Modelos Pedagógicos en la Formación del Profesorado: La Simulación y Juego y El Flipped Classroom. Teoría de la Educación. Educación y Cultura en la Sociedad de la Información,16-30.

Bergmann, J. y Sams, A. (2012). Flip Your Classroom: Reach Every Student in Every Class Every Day. Virginia: ASCD.

Bergmann, J. y Sams, A. (2014). Flipped Learning: Gateway to Student Engagement. ISTE.

Calvillo Castro, A.J. (2014). El modelo Flipped Learning aplicado a la materia de música en el cuarto curso de Enseñanza Secundaria Obligatoria: una investigación-acción para la mejora de la práctica docente y del rendimiento académico del alumnado. (Tesis doctoral). Recuperado de: https://www.educacion.gob.es/teseo/mostrarRef.do?ref=1118301

Campbell, D.T. y Stanley, J.C. (1963). Experimental and quasi-experimental designs for research on teaching. En N.L. Gage (Ed.), Handbook of research teaching. Chicago, IL: Rand McNally.

Chamorro, M. C. (coord.). (2008). Didáctica de las Matemáticas. Madrid: Editorial Pearson. ISBN: 978-84-205-4807-4.

Chickering, A. W. y Gamson, Z. F. (1991). Applying the seven principles for good practice in undergraduate education. San Francisco, CA: Jossey-Bass.

Felder, R. M., Woods, D. R, Stice, J. E y Rugarcia, A. (2000). The future of engineering education: II. Teaching methods than work. Chemical Engineering Education, 34(1), 26-39.

FLN, Flipped Learning Network, (2014) The Four pillars of FLIP. Recuperado de http://www.flippedlearning.org/cms/libo7/VA01923112/Centricity/Domain/46/FLIP ha ndout_FNL_Web.pdf

Galway, L. P., Corbett, K. K., Takaro, T. K., Tairyan, K. y Frank, E. (2014). A novel integration of online and flipped classroom instructional models in public health higher education. BMC Medical Education, 14(1), 181.

Íñigo, V. (2016). Flipped Classroom y la adquisición de competencias en la enseñanza universitaria online. Opción, Año 31, No. Especial 5 (2015): 472 - 479

Lin, Y., Zhu, Y., Chen, C., Wang, W., Chen, T., Li, T., ... y Zou, Y. (2017). Facing the challenges in ophthalmology clerkship teaching: Is flipped classroom the answer?. PloS One, 12(4), e0174829. 
Marcey, D. y Brint, M. (2011). Transforming an undergraduate introductory biology course through cinematic lectures and inverted classes: a preliminary assessment of the clic model of the flipped classroom. California Lutheran University, Thousand Oaks, California. Recuperado de: http://www.nabt.org/websites/institution/File/docs/Four Year Section/2012 Proceedings/Marcey \& Brint.pdf

Marlowe, C.A. (2012). The effect of the flipped classroom on student achievement and stress. Defensa del Master de Ciencias. Montana State University. Bozeman, Montana. Recuperado de: http://scholarworks.montana.edu/xmlui/bitstream/handle/1/1790/MarloweC0812.pdf ?sequence $=1$

Marshall, H. W. (July, 2016). Flipping the online learning space. Paper presented at FlipCon 2016, Allen, TX.

Marshall, H.W. \& Rodriguez-Buitrago, C. J. (forthcoming). The synchronous online flipped learning approach - SOFLA. TEIS Newsletter, TESOL International Association. Recuperado de: http://flglobal.org/the-synchronous-online-flipped-learningapproach/

Martín R., D. y Núñez-del-Río, M. C. (2015). Una experiencia flipped classroom en educación superior: la formación del profesorado de secundaria. Investigar con y para la Sociedad. Asociación Interuniversitaria de Investigación Pedagógica (AIDIPE). Cádiz.

Martín R., D. y Santiago Campión, R. (2015) ¿Es el flipped classroom un modelo pedagógico eficaz? Un estudio sobre la percepción de estudiantes de Primaria, ESO y Bachillerato. Comunicación y Pedagogía 285-286. Flipped Classroom.

Martín R., D. y Santiago Campión, R. (2016). Flipped learning en la formación del profesorado de secundaria y bachillerato. Formación para el cambio Contextos Educativos, 1, 117-134. DOI: 10.18172/con.2854.

Martín R., D. y Tourón, J. (en prensa). El enfoque flipped learning en estudios de magisterio: percepción de los alumnos. Revista Iberoamericana de Educación a Distancia (RIED).

O'Flaherty, J. y Phillips, C. (2015). The use of flipped classrooms in higher education: A scoping review. The Internet and Higher Education, 25, 85-95.

Pierce, R. y Fox, J. (2012). Vodcasts and active-learning exercises in a "flipped classroom" model of a renal pharmacotherapy module. American Journal of Pharmaceutical Education, 76(10), 196.

Prieto, A., Díaz, D., Monserrat, J. y Reyes, E. (2014).Experiencias de aplicación de estrategias de gamificación a entornos de aprendizaje universitario. ReVisión, 7(2).

Quirk-Dorr, D., Anderson, J. y Hoover, W. (2013). Flipping the Classroom Panel Discussion. McGraw-Hill Higher Education. Recuperado de https://www.youtube.com/watch?v=3T8jfzCJzao

Rivera P., Sánchez P., Romo E., Jaramillo A y Valencia A. (2013). Percepciones de los estudiantes universitarios frente al aprendizaje por medio de dispositivos móviles. Revista de Educación y Desarrollo Social. 7(2), 152-165.

Rué, J. (2007). Enseñar en la Universidad: El EEES como reto para la Educación Superior. Madrid: Narcea Ediciones. 
Szoka, J (2013). Measured Results Demonstrate Enhanced Learning Outcomes in the Flipped Classroom. Recuperado de http://www.emergingedtech.com/2013/05/measuredresults-demonstrate-enhanced-learning-outcomes-in-the-flipped-classroom/

Sun, J. C. Y., Wu, Y. T. y Lee, W. I. (2016). The effect of the flipped classroom approach to OpenCourseWare instruction on students' self-regulation. British Journal of Educational Technology.

Thai, T. N., De Wever, B. y Valcke, M. (2017). The impact of a flipped classroom design on learning performance in higher education: Looking for the best "blend" of lectures and guiding questions with feedback. Computers \& Education, 107,113-126

Tourón, J. (24 de mayo de 2016). ¿Flipar clases online? Deshaciendo algunos mitos. Recuperado de http://www.javiertouron.es/2016/05/flipar-clases-onlinedeshaciendo.html

Tourón, J. y Santiago, R. (2015). El modelo Flipped Learning y el desarrollo del talento en la escuela. Revista de Educación, 368, 196-231.

Tourón, J., Santiago, R. y Díez, A. (2014). The Flipped Classroom: Cómo convertir la escuela en un espacio de aprendizaje (Innovación educativa). Grupo Océano

Valero, C. C., Redondo, M. R. y Palacín, A. S. (2012). Tendencias actuales en el uso de dispositivos móviles en educación. La Educación Digital Magazine, 147, 1-21.

Valiente Lopez, M., Kindelan, P., Ayuga Téllez, E. y Martin Rodriguez, D. (2015) Design of a tool to assess the perception of university professors on the implementation of the bologna process: validation by experts. Facing the Challenges of Education. Actas Congreso ICERI.

Walsh, K. (2013a). Flipped Classroom Panel Discussion Provides Rich Insights into a Powerful Teaching Technique. Recuperado de http://www.emergingedtech.com/2013/06/flipped-classroom-panel-discussionprovides-rich-insights-into-a-powerful-teaching-techniquel

Walsh, K. (2013b). Gathering Evidence that Flipping the Classroom can Enhance Learning Outcomes. Recuperado de http://www.emergingedtech.com/2013/03/gatheringevidence-that-flipping-the-classroom-can-enhance-learning-outcomes/ 


\section{Anexo I. Preguntas Cuestionario de satisfacción}

Mis resultados de aprendizaje se han incrementado

Me divierto aprendiendo

Dispongo de mayor autonomía de mi aprendizaje

He mejorado mi proceso de aprendizaje

He trabajado más mi expresión oral o escrita

He incrementado mi creatividad

He aumentado mi motivación

He podido autoevaluar mi proceso de aprendizaje

El profesor dispone un clima facilitador de aprendizaje

He utilizado tecnología digital para aprender

Esta metodología me ha gustado más que la "tradicional"

La inversión en tiempo ha sido mayor que con una metodología tradicional

El profesor tiene más en cuenta mis puntos fuertes, debilidades e intereses

Creo que el aprendizaje es más activo y experiencial

He tenido más posibilidades de participar en la resolución de problemas y desarrollar mi pensamiento crítico

He participado más en la toma de decisiones al colaborar con otros compañeros

Veo más posibilidades para mostrar, al profesor o a mis compañeros, lo que he aprendido

Tengo más posibilidades de trabajar a mi propio ritmo

He tenido facilidad para acceder a los materiales y contenidos de aprendizaje

Mis interacciones con los compañeros durante la clase han sido más positivas

Mis interacciones con el profesor durante la clase han sido más positivas

Mis interacciones con el profesor durante la clase han sido más frecuentes 